\title{
10 Bold Steps to Prevent Burnout in General Internal Medicine
}

\author{
Mark Linzer, $M D^{7,2}$, Rachel Levine, MD, $M P H^{3}$, David Meltzer, MD, $P h D^{4}$, Sara Poplau, $B A^{7}$, \\ Carole Warde, MD ${ }^{5}$, and Colin P. West, MD, PhD ${ }^{6}$
}

'Division of General Internal Medicine, Hennepin County Medical Center, Minneapolis, MN, USA; ${ }^{2}$ University of Minnesota, Minneapolis, MN, USA; ${ }^{3}$ Johns Hopkins University School of Medicine, Baltimore, MD, USA; ${ }^{4}$ University of Chicago, Chicago, IL, USA; 5 Greater Los Angeles VA Health System and UCLA, Los Angeles, CA, USA; ${ }^{6}$ Mayo Clinic, Rochester, MN, USA.

KEY WORDS: burnout; prevention; general internal medicine.

J Gen Intern Med 29(1):18-20

DOI: $10.1007 / \mathrm{s} 11606-013-2597-8$

(C) Society of General Internal Medicine 2013

$\mathrm{P}$ hysician burnout is an escalating problem receiving little attention from health care leaders. Burnout is a long-term stress reaction which includes emotional exhaustion, depersonalization, and a lack of sense of personal accomplishment. Physician burnout rates range from $30-65 \%$ across medical specialties, with the highest rates experienced by those at the front line of care, including emergency medicine and general internal medicine. ${ }^{1}$ Recruitment of medical students into general internal medicine is worrisomely low, and may relate in part to the perceived stressfulness of a primary care career. Addressing burnout among general internists is a national imperative, as health care reform necessitates greater reliance on primary care.

Burnout poses problems for both health care organizations and patients. While burned-out physicians attempt to maintain quality of care at their own expense, work conditions that result in burnout are associated with poorer care quality. ${ }^{2}$ Burned out doctors are more likely to leave their practice, thus reducing access to care. Turnover sacrifices continuity, and replacement costs are at least $\$ 250,000$ per primary care physician. Satisfied role models could make a difference in this steady drain on primary care, but it is getting harder to find them. The situation may be no better for hospitalist physicians, for whom burnout is also common. ${ }^{3}$ Women physicians in national surveys have a $60 \%$ higher burnout rate than that seen in men, yet the workplace remains largely unmindful of gender as a predictor of burnout. And despite known work condition challenges that contribute to burnout in clinics serving minority patients, few if any changes have occurred to improve this situation. Finally, models to prevent physician burnout are not well documented.

Presented in part at a symposium, "Off the treadmill: preventing burnout in General Internal Medicine" at the annual meetings of the Society of General Internal Medicine, Denver, CO-April 262013.

Published online September 4, 2013
We offer suggestions for addressing these challenges. The fact that burnout is a long-term stress reaction allows time to measure and intervene. To combat burnout, organizations need to identify stress in its earlier stages (Fig. 1), and choose programs to prevent burnout before it occurs. Following this quality improvement (QI) model for organizational self-care can produce a sustainable workplace for clinicians, with high quality and accessible care for patients (see Table 1).

\section{INSTITUTIONAL METRICS}

1. Ensure that metrics for institutional success include physician satisfaction and well-being. Any system that does not measure, monitor and optimize clinician wellbeing and sustainability is at risk. We suggest measuring the following metrics: predictors (e.g., work control, time pressure, pace of work [chaos] and values alignment between clinicians and leaders), and outcomes (e.g., physician satisfaction, stress, burnout and intent to leave the practice). Tools for these measures and their relationships with quality of care have previously been published $^{1,2}$ and short measurement tools are in development. Utilizing data in a continuous QI model would allow practices to address predictors of poor clinician outcomes and drive a decline in burnout rates. One mechanism to accomplish this work is to form a clinician wellness committee such as those at Stanford University led by Dr. Bryan Bohman and within the Permanente Medical Group of Kaiser Permanente directed by Dr. Betty Kung. These committees are staffed with divisional champions from across disciplines. Attention to healthy eating, exercise, and efforts to minimize stress hold great promise for decreasing burnout.

2. Incorporate mindfulness and teamwork for trainees and practicing clinicians. Mindfulness, a known stress reducer, is a means for internally accommodating to external stressors. Teamwork, such as in the Patient-Centered Medical Home (PCMH), is critically important for burnout prevention through support and sharing of clinician workload. Tools 


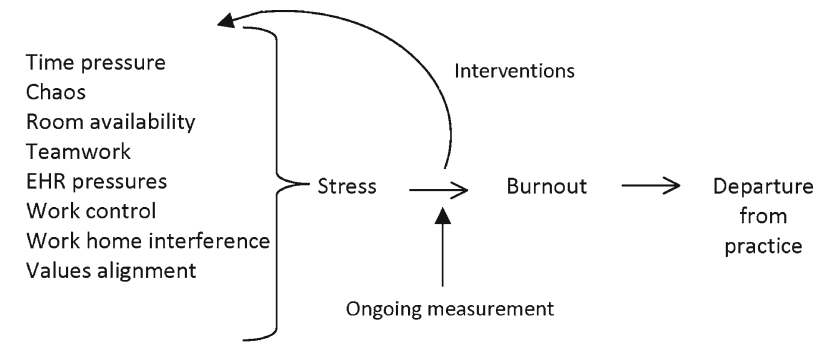

EHR = Electronic Health Record

Figure 1. Conceptual model of QI feedback loop to prevent stress, burnout and turnover.

being developed to measure the clinician experience with the PCMH can assure the model works for current and future physicians. Leaders should assure protected time for reflection, planning and relationship building and provide communication coaching for challenging situations.

3. Decrease stress from electronic health records (EHR). There has been a dramatic increase in the amount of computer work required by primary care physicians. Strategies to address this include changing visit length to accommodate the extra work that computerized data brings, or adding "desktop" slots to daily schedules. Additional stresses have arisen from patient portals which increase after-hours communication. We propose measuring workloads, both direct (with patients) and indirect, as well as stress due to the EHR. These results should be part of an organization's "dashboard" of clinical outcomes.

\section{WORK CONDITIONS}

4. Address challenging work conditions in primary care clinics, especially those serving minority patients. Work conditions are more challenging in clinics serving minority patients. ${ }^{4}$ Improving work conditions in these clinics will require leaders to provide sufficient clinical resources (supplies and equipment), room availability and access to specialists. Indeed, these "work condition quality metrics" will be useful for clinics serving all patients.

5. Cover predictable life events with clinician "float pools." A typical GIM division has $10 \%$ less FTE than anticipated due to predictable life events; ${ }^{5}$ certain scenarios can lead to a shortage of 20-30 \% FTEs. Planning for life events with float pool coverage may be cost effective, as coverage raises morale and avoids burnout and turnover.

6. Develop practice models which preserve physician work control. Many health care organizations are "standardizing" to reduce variations in physician work (e.g., requirements for specific numbers of sessions per week, patients per session, etc.). While standardization may lead to better work role definition, it also reduces flexibility and control. The "demand-control" model of job stress shows that high work demands are mitigated by work control. In research in Swedish non-physician workers, removing control put workers at risk for high stress and adverse cardiovascular outcomes. To preserve work control, standardization must still allow clinicians to "customize" their schedules to allow space for their other clinical and non-clinical activities.

7. Support manageable primary care panel sizes, lengthened visits and enhanced staffing ratios. One PCMH demonstration project in Seattle reduced panel sizes, lengthened visits and supported teamwork through adequate staffing. This was accompanied by a sharp drop in burnout, yet costs were neutral and quality preserved. This suggests that altering staffing ratios may reduce stress and burnout. Leaders in PCMH care have recently called for "sharing the care" with expanded primary care teams. Thus, there is growing support for maintaining reasonable panel sizes and sufficient staff to co-manage these panels with clinicians.

Table 1. Ten Steps to Prevent Physician Burnout

\footnotetext{
Institutional Metrics

1. Make clinician satisfaction and wellbeing quality indicators.

2. Incorporate mindfulness and teamwork into practice.

3. Decrease stress from electronic health records.

Work Conditions

4. Allocate needed resources to primary care clinics to reduce healthcare disparities.

5. Hire physician floats to cover predictable life events.

6. Promote physician control of the work environment.

7. Maintain manageable primary care practice sizes and enhanced staffing ratios.

Career Development

8. Preserve physician "career fit" with protected time for meaningful activities.

9. Promote part-time careers and job sharing.

Self-Care

10. Make self-care a part of medical professionalism
} 


\section{CAREER DEVELOPMENT}

8. Address "career fit", so faculty have time to do what they are passionate about. Mayo Clinic researchers have demonstrated that when academic physicians have less than 10-20\% FTE time to do what they care about most, burnout rates increase to extremely high levels $(>50 \%)$. We propose that it is cost effective to provide at least one half day per week for clinicians to do what they are most passionate about.

Another model of career fit is Stanford's Academic Biomedical Career Customization (ABCC) program, under the leadership of Drs. Hannah Valantine, Caroline Simard and Christy Sandborg. ABCC focuses on: 1) implementing career planning with transparent conversations around work-life needs and 2) reducing worklife conflict by supporting grant editing, presentation preparation and home task outsourcing services.

The unique aspects of hospital based medicine require a targeted approach that incorporates approaches to burnout prevention. Weekend, evening and night hours present unique challenges to worklife balance, while creating opportunities to provide time off during traditional weekday working hours. Successful careers will be more likely in hospitalbased medicine if academic hospitalists are effective in both "systole" (clinical work) and "diastole" (non-clinical work and time off). Balancing systole and diastole may prevent burnout by allowing hospitalists to develop skills and career paths in areas such as education, research and QI.

9. Promote career opportunities and advancement for parttime physicians. Flexible career policies including parttime work and shared medical practices (job sharing) between two physicians are critical for enhancing work/ life balance. In a large study of US physicians, those with the highest work/life balance had the lowest rates of burnout. ${ }^{1}$ Part-time physicians are satisfied with their careers, provide high-quality care, and are less likely to leave their jobs. The gender differences in burnout identified among US physicians are not seen in the Netherlands, where $75 \%$ of women physicians work part-time. Part-time options allow institutions to use a more flexible career life cycle approach to meet the needs of an increasingly diverse workforce and prevent burnout.

\section{SELF-CARE}

10. Prioritize physician self-care as an element of medical professionalism. Professionalism is defined by altruism and compassion in treating others. We propose that professionalism be extended to include how physicians care for themselves. Physician distress negatively impacts both physicians and patients. Adjusting traditional views of the "diligent, hardworking, self-sacrificing" physician to also prioritize physician self-care will take strong leadership.

Implementing these 10 suggestions will help attract learners to careers in general internal medicine and provide sustainability for those who practice it. Healthcare organizations may use these suggestions to begin a process to eliminate burnout. A QI agenda to decrease burnout is in the best interests of all physicians and patients. We invite others to join us in this journey.

Acknowledgements: Financial support for this paper: Dr. David Meltzer is supported by a Robert Wood Johnson Foundation Investigator Award and by the National Institute of Aging through a Midcareer Career Development Award (1 K24 AG031326-01). Dr. Mark Linzer and Ms. Sara Poplau were supported by an Agency for Healthcare Research and Quality grant (1R18HSO18160-01), "Creating Healthy Workplaces."

Conflict of Interest: The authors declare they do not have any conflicts of interest.

Corresponding Author: Mark Linzer, MD; Division of General Internal Medicine, Hennepin County Medical Center, 701 Park Ave, Minneapolis, MN 55415, USA (e-mail: Mark.linzer@hcmed.org).

\section{REFERENCES}

1. Shanafelt TD, Boone S, Tan L, et al. Burnout and satisfaction with worklife balance among US physicians relative to the general US population. Arch Intern Med. 2012;172(18):1377-85.

2. Linzer M, Manwell LB, Williams ES, et al. Working conditions in primary care: physician reactions and care quality. Ann Intern Med. 2009;151:28-36.

3. Meltzer DO. Social science insights into improving workforce effectiveness: examples from the developing field of hospital medicine. J Pub Health Manag Pract. 2009;15(6):S18-23.

4. Varkey AB, Baier Manwell L, Ibrahim SA, et al. Impact of work conditions on errors and quality. J Gen Intern Med. 2013;28(1S):S100.

5. Linzer M, Rosenberg M, McMurray JE, Glassroth J. Respecting the lifecycle: rational workforce planning in a section of general internal medicine. Am J Med. 2002;113:443-48. 